\title{
Correlation of Preoperative D-Dimer Levels and Postoperative Venous Thromboembolism in Patients Undergoing Total Hip Arthroplasty and Total Knee Arthroplasty
}

\author{
Sanjay Agarwala*, Mayank Vijayvargiya, Neeraj Mishra \\ Department of Orthopedics, P.D. Hinduja National Hospital, Mumbai, India \\ Email: *drsa2011@gmail.com
}

How to cite this paper: Agarwala, S., Vijayvargiya, M. and Mishra, N. (2021) Correlation of Preoperative D-Dimer Levels and Postoperative Venous Thromboembolism in Patients Undergoing Total Hip Arthroplasty and Total Knee Arthroplasty. Open Journal of Orthopedics, 11, 269-277. https://doi.org/10.4236/ojo.2021.119025

Received: August 1, 2021

Accepted: September 15, 2021

Published: September 18, 2021

Copyright $\odot 2021$ by author(s) and Scientific Research Publishing Inc. This work is licensed under the Creative Commons Attribution International License (CC BY 4.0).

http://creativecommons.org/licenses/by/4.0/ (c) (i) Open Access

\begin{abstract}
Background: The relationship of preoperative D-dimer and Venous thromboembolism (VTE) after total knee arthroplasty (TKA) and total hip arthroplasty (THA) remains controversial. The purpose of this study was to assess the value of D-dimer in the detection of early VTE after TKA and THA. Materials and Methods: A total of 312 patients were evaluated in this retrospective study from 2016 to 2020 at a tertiary care centre, Mumbai who were operated for THA and TKA. The measurement of plasma D-dimer level was done preoperatively and clinically symptomatic patients were evaluated and confirmed for VTE by Colour Doppler Sonography. The plasma D-dimer levels were correlated statistically with the sonographic VTE. Results: 11 patients developed Deep Vein Thrombosis (DVT) and 1 patient developed Pulmonary embolism (PE). Preoperative plasma D-dimer levels were significantly higher $(\mathrm{P}<0.01)$ in patients who developed DVT postoperatively. Colour Doppler Sonography showed that 2 thrombi were seen in proximal veins (pelvic and femoral veins), and 2 thrombi were seen in distal veins (posterior tibial, peroneal, gastrocnemian veins) of the calf in THA patients, and all 8 thrombi were seen in the distal veins (posterior tibial, peroneal, gastrocnemian veins) of the calf in TKA patients. Conclusion: We infer that if the preoperative D-dimer levels were more than $0.5 \mathrm{mg} / \mathrm{l}$, the chances of developing VTE postoperatively are more, which can be diagnosed clinically and further by other investigations. In this study, we have shown that doing preoperative D-dimer level can almost PREDICT the development of VTE and thus prevent complications.
\end{abstract}

\section{Keywords}

Plasma D-Dimer, DVT, Colour Doppler, Clinical DVT, Preoperative 


\section{Introduction}

Anticipating medical disasters and reducing medical challenges has been considered in the realms of Astrologers and Soothsayers. Venous thromboembolism (VTE) is a complex vascular disease that commonly manifests as deep vein thrombosis (DVT) and pulmonary embolism (PE) [1]. Virchow's concept of thrombosis involves a triad of factors, namely hypercoagulability, venous stasis, and endothelial injury [2]. VTE is commonly seen in post arthroplasty patients of hip and knee in patients who do not receive chemoprophylaxis postoperatively [3].

A study in California of 43,000 people over four years reported incidence of VTE to be $2.1 \%$ after THA and $2.8 \%$ after TKA [4]. In the Indian population, the incidence of early DVT was $52.1 \%$, and specifically, the incidence of DVT in THA patients was $9.1 \%$, and TKA patients were $4.1 \%$ [5].

The aetiology of DVT is multifactorial, and includes conditions like Surgery or Trauma, Pregnancy, Malignancy, prolonged immobilization, Congestive heart failure, obesity, advancing age, and Idiopathic [6]. Various cultural and ethnic factors are also responsible for the higher rates of DVT in Asian and Indian populations, as an increase in life expectancy, dietary problems with increased fat and carbohydrate intake and sedentary lifestyle issues [5] [7] [8] [9] [10].

The role of D-dimer to predict the occurrence of DVT is controversial. There is a lack of evidence about the correlation of preoperative D-dimer levels and postoperative VTE in the patients of the Indian subcontinent [11]-[17].

The plasma D-dimer level is a good indicator of VTE in high-risk patients. $\mathrm{D}$-dimer is formed when fibrinolytic like plasmin degrades cross-linked fibrin strands [11] [12]. D-dimer can be tested by ELISA, latex photometry and immunoturbidimetric. Various studies have found levels of D-dimer to be elevated in patients developing VTE postoperatively [13] [14] [15] [16]. Our hypothesis is that the D-dimer levels can be used to predict the onset of VTE after THA and TKA [13] [14].

The current study is a retrospective study from 2016 to 2020 wherein D-dimer levels were routinely performed preoperatively in patients undergoing THA and TKA, and the patients were followed postoperative clinically for the presence of symptoms and signs of VTE.

In this study, we present convincing evidence of a simple D-dimer blood test as a part of the routine preoperative profile for surgical fitness as a predictive test for Venous Thromboembolism and thus mitigating morbidity and mortality of operative patients.

\section{Materials and Methods}

\subsection{Study Population}

From the year 2016 to 2020 , a total of 384 patients were operated on for TKA 
and THA, wherein 72 patients who had a history of DVT were under ongoing treatment of DVT and were at high risk for DVT (congestive heart failure, stroke, myocardial infarction, malignancy, chronic renal and hepatic conditions, APLA syndrome, coagulopathies, etc.) have been excluded from this analysis.

Hence a total of 312 patients were finally evaluated, out of which 113 were operated for THA, and 199 were operated for TKA. In the THA group, 71 were male, and 42 were female patients, the average age being 57.27 years (range 25 87 years). 106 patients were primary THA, and 7 were revision THA. The indications for surgery in this group included Avascular necrosis of the hip in 89, Osteoarthritis in 11, Rheumatoid arthritis in 10 and Transcervical neck femur fracture in 3 patients.

In the TKA group, 41 were males, and 158 were females, with an average age of 66.1 years (range 44 - 78 years). All the TKA patients were undergoing primary TKA, and the condition for surgery in this group included Osteoarthritis in 168 and rheumatoid arthritis in 31 patients.

\subsection{Preoperative Investigations}

Prior to admission, all the patients routinely had complete blood count, serum electrolytes, liver function tests, renal function tests, blood sugar levels, MRSA screening (nasal and axilla swab), anti-HIV, anti HCV, anti-HBsAg, blood grouping, cross-matching, Coagulation profile and D-dimer levels. A physician/cardiologist/anaesthetist approval was taken for fitness for surgery.

D-dimer levels were checked using Immunometric method which is similar to ELISA technique and is a good quantitative method with high sensitivity [17]. The D-dimer levels were defined as negative $(<0.25 \mathrm{~g} / \mathrm{l})$, minimal $(0.25-0.5$ $\mathrm{mg} / \mathrm{l})$, low (0.5 - $1.0 \mathrm{mg} / \mathrm{l})$, medium (1.0 - $2.0 \mathrm{mg} / \mathrm{l})$ and high (>2.0 mg/l) [17].

The demographic data, duration of surgery and medical comorbidities like diabetes mellitus, hypertension were also recorded. All the surgeries were performed by the senior author at this institution.

All TKA surgeries were performed under a tourniquet. All patients received subcutaneous injection Dalteparin 5000 IU once daily for 14 days in TKA and 35 days in THA patients as postoperative thromboprophylaxis [18]. All patients were given mechanical thromboprophylaxis in the form of compression socks and pneumatic compression devices postoperatively. All patients were mobilized full weight-bearing with a walker from the same day of surgery.

\subsection{Assessment}

Postoperatively all patients were evaluated on day 10, 3 weeks, 6 weeks, 3 months, 6 months and 1 year. Patients who complained of pain or swelling in the calf were asked to get a Colour Doppler of the lower limb. Any patient who complained of chest pain and shortness of breath were also evaluated by a physician and were asked to get a Ventilation-Perfusion (V/Q) scan and Computerized Tomography Pulmonary Angiography (CTPA) as and when required. Demo- 
graphic data, duration of surgery (in minutes) and medical comorbidities were also evaluated as a predictive factor VTE.

Preoperative D-dimer levels of all the patients were recorded along with demographic details and duration of surgery, hypertension, diabetes mellitus and the occurrence of VTE. The association between high preoperative D-dimer value with the occurrence of VTE was then analyzed.

\subsection{Statistical Analysis}

Comparison between patients with or without DVT was performed using Independent T-test, Mann Whitney U test, Chi-square values. Cut off values were chosen based on optimal sensitivity and negative predictive value. For all tests, a two-tailed $\mathrm{P}$ value $<0.01$ was considered to be statistically significant.

\section{Results}

A total of 12 patients out of 312 patients included in this study group developed symptomatic VTE (3.84\%), out of which 11 patients had DVT (3.52\%), and 1 patient developed pulmonary embolism $(0.32 \%)$. Association of Age, Gender, hypertension, diabetes mellitus, duration of surgery, varicose veins with the risk of VTE was found to be statistically not significant. Other factors like low platelet count, Body Mass Index (BMI) were also not statistically significant. None of the patient who developed clinical DVT in the study needed post operative ICU stay (Table 1 and Table 2).

4 patients out of 199 patients in the TKA group and 7 patients out of 113 patients developed DVT. Although there was no statistically significant association seen with the occurrence of DVT with the type of surgery (THA, TKA), although we have seen that DVT was more common in the THA group (Table 3).

Preoperatively D-dimer levels were raised in 56 patients, and postoperatively DVT developed in 11 patients, out of whom 5 were male, and 6 were female. 10 out of 11 patients who developed DVT in our series had high D-dimer levels. 1 patient developed clinical DVT with D-dimer levels was less than $0.5 \mathrm{mg} / \mathrm{l}$ (low or normal range). 1 female patient had developed Pulmonary embolism despite having a normal or low D-dimer level (i.e. $<0.5 \mathrm{mg} / \mathrm{l}$ ).

The above was examined by colour Doppler sonography after the patient was clinically symptomatic.

After the patients developed clinical DVT which was confirmed by Doppler sonography, the patient was sent for a cardiologist and physician opinion for starting the treatment. The treatment consisted of injectable and oral anticoagulant therapy along with pneumatic compression devices for mechanical thromboprophylaxis. No fatalities were observed were observed in this study and all patients were discharged from the hospital after two weeks of hospital stay.

\section{Discussion}

Venous thromboembolism was one of the leading causes of morbidity and 
Table 1. Patients demographics.

\begin{tabular}{|c|c|c|c|c|c|c|}
\hline \multirow{2}{*}{ Category } & \multicolumn{2}{|c|}{ DVT } & \multirow{2}{*}{ Total } & \multirow{2}{*}{$\begin{array}{l}\text { Chi-square } \\
\text { Value }\end{array}$} & \multirow{2}{*}{$\mathrm{df}$} & \multirow{2}{*}{$\begin{array}{l}\text { Exact } \\
\text { P-value }\end{array}$} \\
\hline & Present & Absent & & & & \\
\hline \multicolumn{7}{|c|}{ Age (in yrs) } \\
\hline \multirow{2}{*}{ Upto 30} & 0 & 12 & 12 & \multirow{10}{*}{1.911} & \multirow{10}{*}{3} & \multirow{10}{*}{0.546} \\
\hline & $0.0 \%$ & $4.0 \%$ & $3.8 \%$ & & & \\
\hline \multirow{2}{*}{$31-45$} & 1 & 20 & 21 & & & \\
\hline & $9.1 \%$ & $6.6 \%$ & $6.7 \%$ & & & \\
\hline \multirow{2}{*}{$46-60$} & 1 & 71 & 72 & & & \\
\hline & $9.1 \%$ & $23.6 \%$ & $23.1 \%$ & & & \\
\hline \multirow{2}{*}{$>60$} & 9 & 198 & 207 & & & \\
\hline & $81.8 \%$ & $65.8 \%$ & $66.3 \%$ & & & \\
\hline \multirow{2}{*}{ Total } & 11 & 301 & 312 & & & \\
\hline & $100.0 \%$ & $100.0 \%$ & $100.0 \%$ & & & \\
\hline \multicolumn{7}{|l|}{ Sex } \\
\hline \multirow{2}{*}{ Male } & 5 & 107 & 112 & \multirow{6}{*}{0.453} & \multirow{6}{*}{1} & \multirow{6}{*}{0.532} \\
\hline & $45.5 \%$ & $35.5 \%$ & $35.9 \%$ & & & \\
\hline \multirow{2}{*}{ Female } & 6 & 194 & 200 & & & \\
\hline & $54.5 \%$ & $64.5 \%$ & $64.1 \%$ & & & \\
\hline \multirow{2}{*}{ Total } & 11 & 301 & 312 & & & \\
\hline & $100.0 \%$ & $100.0 \%$ & $100.0 \%$ & & & \\
\hline \multicolumn{7}{|l|}{ DM } \\
\hline \multirow{2}{*}{ Present } & 5 & 117 & 122 & \multirow{6}{*}{0.193} & & \\
\hline & $45.5 \%$ & $38.9 \%$ & $39.1 \%$ & & & \\
\hline Abent & 6 & 184 & 190 & & 1 & 0756 \\
\hline 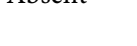 & $54.5 \%$ & $61.1 \%$ & $60.9 \%$ & & 1 & 0.750 \\
\hline $\mathrm{T}$ & 11 & 301 & 312 & & & \\
\hline 10lut & $100.0 \%$ & $100.0 \%$ & $100.0 \%$ & & & \\
\hline HTN & & & & & & \\
\hline Precant & 7 & 133 & 140 & & & \\
\hline FItesent & $63.6 \%$ & $44.2 \%$ & $44.9 \%$ & & & \\
\hline Ahent & 4 & 168 & 172 & 1623 & 1 & 0230 \\
\hline НUSEIII & $36.4 \%$ & $55.8 \%$ & $55.1 \%$ & $1.02 J$ & 1 & 0.230 \\
\hline Tetol & 11 & 301 & 312 & & & \\
\hline 10tat & $100.0 \%$ & $100.0 \%$ & $100.0 \%$ & & & \\
\hline Type of Su & & & & & & \\
\hline TKR & 4 & 195 & 199 & & & \\
\hline & $36.4 \%$ & $64.8 \%$ & $63.8 \%$ & & & \\
\hline TUD & 7 & 106 & 113 & & & \\
\hline $1 \mathrm{HK}$ & $63.6 \%$ & $35.2 \%$ & $36.2 \%$ & 3.711 & 1 & 0.063 \\
\hline Toth & 11 & 301 & 312 & & & \\
\hline & $100.0 \%$ & $100.0 \%$ & $100.0 \%$ & & & \\
\hline
\end{tabular}


Table 2. Relation with duration of surgery and age.

\begin{tabular}{ccccccc}
\hline & DVT & N & $\begin{array}{c}\text { Mean } \\
\text { D-DIMER }\end{array}$ & $\begin{array}{c}\text { Std. } \\
\text { Deviation }\end{array}$ & t-value & p-value \\
\hline \multirow{2}{*}{ AGE } & Present & 11 & 67.91 & 15.28 & 1.247 & 0.213 \\
& Absent & 301 & 62.72 & 13.49 & & \\
\hline \multirow{2}{*}{ DURATION IN } & Present & 11 & 59.27 & 5.73 & 0.257 & 0.797 \\
MINUTES & Absent & 301 & 58.73 & 6.86 & & \\
\hline
\end{tabular}

Table 3. Incidence of DVT in TKA and THA.

\begin{tabular}{ccccccc}
\hline Surgery & DVT & N & $\begin{array}{c}\text { Mean } \\
\text { D-DIMER }\end{array}$ & $\begin{array}{c}\text { Std. } \\
\text { Deviation }\end{array}$ & t-value & p-value \\
\hline \multirow{2}{*}{ TKR } & Present & 4 & 1.35 & 1.70 & 1.425 & 0.156 \\
& Absent & 195 & 0.39 & 1.33 & & \\
\hline \multirow{2}{*}{ THR } & Present & 7 & 4.49 & 3.50 & 6.023 & $<0.001$ \\
& Absent & 106 & 0.55 & 1.51 & & \\
\hline
\end{tabular}

mortality in patients who went for arthroplasty surgeries. However, due to recent advances, we have been able to limit the mortality caused by VTE, but the morbidity continues. To reduce the morbidity, we need to make an early diagnosis and prompt treatment of the condition. A battery of pre and postoperative tests are available to diagnose VTE and prevent its complications. One of the tests is the Plasma D-dimer levels which are a very cost-effective test costing around 10 US dollars (around 1000 Indian Rupees).

Various assay systems are available to measure the plasma D-dimer levels. ELISA technique provides the best quantitative analysis of the D-dimer levels, although it is time-consuming and requires skilled technique and is difficult to use for emergency cases. The latex agglutination test is a simple and rapid diagnostic test, but it is less sensitive and tends to underestimate the D-dimer levels. Preoperatively plasma D-dimer levels were evaluated for all patients who were being operated on for arthroplasty of the hip and knee.

In our study, we have used the immunometric method to detect the plasma D-dimer levels, which is similar to the ELISA technique and equally sensitive. The mean D-dimer level was $0.54 \mathrm{mg} / \mathrm{l}$ with a standard deviation of 1.58 and the lower limit within $95 \%$ confidence interval was 0.37 , and the upper limit within $95 \%$ confidence interval was 0.72 . The minimum value of $\mathrm{D}$-dimer in the study group was $0.06 \mathrm{mg} / \mathrm{l}$, and the maximum value was $16.25 \mathrm{mg} / \mathrm{l}$ (Table 4).

In our study, we have found a higher correlation of raised D-dimer levels with the occurrence of DVT. These findings are corroborative with the findings by Shiota et al. There are a few studies like Thunainit C et al. [19], and Bounameaux et al. [20] has said that there is no correlation between preoperative D-dimer levels and postoperative VTE. In our study, it is seen that plasma D-dimer levels can be very reliable and help in the early diagnosis of VTE. This discrepancy might be due to different sample sizes and different techniques of measurement 
Table 4. D-Dimer analysis.

\begin{tabular}{lcc}
\hline \multicolumn{1}{c}{ Statistics for D DIMER } & Value \\
\hline Mean & LL & 0.54 \\
SD & UL & 1.58 \\
95\% Confidence Interval for Mean & & 0.37 \\
Median & 0.72 \\
Minimum & 0.10 \\
Maximum & 0.06 \\
Interquartile Range & 16.25 \\
Skewness & 0.20 \\
Kurtosis & 6.68 \\
\hline
\end{tabular}

of plasma levels of D-dimer.

Clarke et al. [21] reported that the prevalence of DVT was 32\% (16\% proximal, 16\% distal) in THA and 66\% (16\% proximal, 50\% distal) in TKA according to unilateral venography. Fujita et al. [7] used unilateral venography and showed that the occurrence of DVT was $22.6 \%$ (9.8\% proximal, $12.8 \%$ distal) in THA and $48.6 \%$ (14.5\% proximal, $34.1 \%$ distal) in TKA. In our study, we used colour Doppler ultrasonography and found out the prevalence of DVT was 3.5\% $(1.75 \%$ proximal, $1.75 \%$ distal) in THA and $4.04 \%$ (all in distal) in TKA according to colour doppler ultrasonography.

\section{Limitations of Study}

The limitations in our study are that it is small sample size, retrospective in nature, and not all patients were evaluated with Colour Doppler Sonography, but only clinically symptomatic patients were evaluated with colour doppler.

\section{Conclusions}

Our study shows a direct correlation between preoperative D-dimer levels and postoperative development of VTE in the form of DVT. If the preoperative $\mathrm{D}$-dimer levels are more than $0.5 \mathrm{mg} / \mathrm{l}$, there are chances of developing VTE in postoperative patients, which can be diagnosed clinically and further by other investigations.

According to our study, plasma D-dimer levels, when done preoperatively in patients, cannot just be reliable but also predictive of VTE and help in preventing its complications, and thus help in saving a limb or a life in a patient.

\section{Conflicts of Interest}

The authors declare no conflicts of interest regarding the publication of this paper. 


\section{References}

[1] Moser, K.M., Fedullo, P.F., LitteJohn, J.K. and Crawford, R. (1994) Frequent Asymptomatic Pulmonary Embolism in Patients with Deep Venous Thrombosis. JAMA, 271, 223-225. https://doi.org/10.1001/jama.1994.03510270069042

[2] Kumar, D.R., Hanlin, E., Glurich, I., Mazza, J.J. and Yale, S.H. (2010) Virchow's Contribution to the Understanding of Thrombosis and Cellular Biology. Clinical Medicine \& Research, 8, 168-172. https://doi.org/10.3121/cmr.2009.866

[3] Cordell-Smith, J.A., Williams, S.C., Harper, W.M. and Gregg, P.J. (2004) Lower Limb Arthroplasty Complicated by Deep Venous Thrombosis. Prevalence and Subjective Outcome. The Journal of Bone and Joint Surgery. British Volume, 86, 99. https://doi.org/10.1302/0301-620X.86B1.14507

[4] White, R.H., Romano, P.S., Zhou, H., Rodrigo, J. and Bargar, W. (1998) Incidence and Time Course of Thromboembolic Outcomes Following Total Hip or Knee Arthroplasty. Archives of Internal Medicine, 158, 1525-1531. https://doi.org/10.1001/archinte.158.14.1525

[5] Agarwala, S., Bhagat, A., Modhe, J., Dastur, F.D. and Patil, S. (2003) Incidence of Deep Vein Thrombosis in Indian Patients: A Prospective Study in 104 Patients. Indian Journal of Orthopaedics, 37, 159-162.

[6] Mammen, E.F. (1992) Pathogenesis of Venous Thrombosis. Chest, 102, 640S-644S. https://doi.org/10.1378/chest.102.6 Supplement.640S

[7] Fujita, S., Hirota, S., Oda, T., Kato, Y., Tsukamoto, Y. and Fuji, T. (2000) Deep Venous Thrombosis after Total Hip or Total Knee Arthroplasty in Patients in Japan. Clinical Orthopaedics and Related Research, 375, 168. https://doi.org/10.1097/00003086-200006000-00020

[8] Ko, P.S., Chan, W.F., Siu, T.H., Khoo, J., Wu, W.C. and Lam, J.J. (2003) Deep Venous Thrombosis after Total Hip or Knee Arthroplasty in a "Low-Risk" Chinese Population. The Journal of Arthroplasty, 18, 174-179. https://doi.org/10.1054/arth.2003.50040

[9] Leizorovicz, A., Turpie, A.G., Cohen, A.T., Pellois, A., Diebolt, P. and Darmon, J.Y. (2004) Epidemiology of Post-Operative Venous Thromboembolismm in Asian Countries. International Journal of Angiology, 13, 101-108.

https://doi.org/10.1007/s00547-004-1010-1

[10] Maini, P.S., Talwar, N., Nijhawan, V.K. and Dhawan, M. (2006) Results of Cemented Bipolar Hemiarthroplasty for Fracture of the Femoral Neck-10 Year Study. Indian Journal of Orthopaedics, 40, 154-156.

https://doi.org/10.4103/0019-5413.34481

[11] Waser, G., Kathriner, S. and Wuillemin, W.A. (2005) Performance of the Automated and Rapid STA Liatest D-Dimer on the STA-R Analyzer. Thrombosis Research, 116, 165. https://doi.org/10.1016/j.thromres.2004.12.003

[12] Wells, P.S., Anderson, D.R., Rodger, M., Forgie, M., Kearon, C., Dreyer, J., Kovacs, G., Mitchell, M., Lewandowski, B. and Kovacs, M.J. (2003) Evaluation of D-Dimer in the Diagnosis of Suspected Deep-Vein Thrombosis. The New England Journal of Medicine, 349, 1227-1235. https://doi.org/10.1056/NEJMoa023153

[13] Dunn, I.D., Hui, A.C., TriYtt, P.D., Crozier, A.E., Gregg, P.J., Sinclair, M.E., Armstrong, A.L. and Mitchell, V.E. (1994) Plasma D-Dimer as a Marker for Postoperative Deep Venous Thrombosis. A Study after Total Hip or Knee Arthroplasty. Thrombosis and Haemostasis, 72, 663-665. https://doi.org/10.1055/s-0038-1648938

[14] Dale, S., Gogstad, G.O., Brosstad, F., et al. (1994) Comparison of Three D-Dimer 
Assays for the Diagnosis of DVT: ELISA, Latex and an Immunofiltration Assay (NycoCard D-Dimer). Thrombosis and Haemostasis, 71, 270-274. https://doi.org/10.1055/s-0038-1642428

[15] Legnani, C., Pancani, C., Palareti, G., et al. (1997) Comparison of New Rapid Methods for D-Dimer Measurement to Exclude Deep Vein Thrombosis in Symptomatic Outpatients. Blood Coagulation \& Fibrinolysis, 8, 296-302.

https://doi.org/10.1097/00001721-199707000-00006

[16] Meissner, M.H., Zierler, B.K., Bergelin, R.O., et al. (2000) Markers of Plasma Coagulation and Fibrinolysis after Acute Deep Venous Thrombosis. Journal of Vascular Surgery, 32, 870-880. https://doi.org/10.1067/mva.2000.110359

[17] Shiota, N., Sato, T., Nishida, K., Matsuo, M., Takahara, Y., Mitani, S., Murakami, T. and Inoue, H. (2002) Changes in LPIA D-Dimer Levels after Total Hip or Knee Arthroplasty Relevant to Deep-Vein Thrombosis Diagnosed by Bilateral Ascending Venography. Journal of Orthopaedic Science, 7, 444-450. https://doi.org/10.1007/s007760200077

[18] Won, Y., Kim, M., Jun, K., Nam, W., Ahn, S., Hwang, J.-K., Kim, S.-D., Park, S.-C., Yun, S., Lee, W.-C., Park, J.-S. and Kim, J. (2014) Incidence and Clinical Characteristics of Deep Vein Thrombosis (DVT) after Total Knee Arthroplasty (TKA) with DVT Chemoprophylaxis. World Journal of Cardiovascular Diseases, 4, 531-538. https://doi.org/10.4236/wjcd.2014.411064

[19] Chotanaphuthi, T., Heebthamai, D., Taweewuthisub, W., Thiengwittayaporn, S., Roschan, S. and Kanchanaroek, K. (2009) Prediction of Deep Vein Thrombosis after Total Knee Arthroplasty with Preoperative D-Dimer Plasma Measurement. Journal of the Medical Association of Thailand, 92, S6-S10.

[20] Bounameaux, H., Miron, M.J., Blanchard, J., et al. (1998) Measurement of Plasma D-Dimer Is Not Useful in the Prediction or Diagnosis of Postoperative Deep Vein Thrombosis in Patients Undergoing Total Knee Arthroplasty. Blood Coagulation \& Fibrinolysis, 9, 749-752. https://doi.org/10.1097/00001721-199811000-00004

[21] Clarke, M.T., Green, J.S., Harper, W.M., et al. (1997) Screening for Deep Venous Thrombosis after Hip and Knee Replacement without Prophylaxis. The Journal of Bone and Joint Surgery. British Volume, 79, 787-791. https://doi.org/10.1302/0301-620X.79B5.0790787 